\title{
Littéracie, apprentissage et enseignement pour tous
}

Dans ces actes du colloque IAIMTE (International Association for the Improvement of Mother Tongue Education) qui a eu lieu à l'université Paris Est Créteil en juin 2013, nous nous intéressons tout particulièrement aux questions d'enseignement apprentissage en lien avec la litéracie. Les résultats des évaluations des élèves à l'échelle internationale (PISA pour les élèves de 15 ans, PIRLS pour les élèves de la quatrième de l'école élémentaire) ont retenu l'attention des décideurs, des enseignants et des chercheurs dans deux domaines fondamentaux:

- La différence entre les systèmes et les pratiques qui concernent l'amélioration des compétences litéraciées.

- Les différences entre les systèmes et les pratiques au regard du principe d'équité : dans certaines situations, l'écart entre élèves peut s'accroître alors que dans d'autres, les pratiques enseignantes peuvent améliorer les résultats des élèves les plus performants, comme les plus faibles.

Ces résultats éclairent également les différentes manières qui conduisent à définir les notions d'efficacité et d'équité, en fonction des pays.

Nous nous focalisons plus particulièrement sur l'enseignement et l'apprentissage effectifs, sur les niveaux atteints, ou devant être atteints, par les élèves, les enseignants, les écoles et le système éducatif dans son ensemble.

Ainsi les auteurs des articles ci-dessous ont interrogé les manières d'enseigner et d'apprendre :

- Comment lire, comment écrire et comment parler de l'effet de la litéracie à l'école en termes d'efficacité et d'équité ?

- Quel écart entre les résultats attendus des élèves à chaque stade de leur scolarité et ce qui est effectivement enseigné ? Entre enseignement prescrit et enseignement réel ?

- Comment les enseignants ou le système éducatif accompagne(nt) les élèves pour leur permettre d'atteindre les objectifs définis dans les programmes ?

Les chercheurs se sont intéressés aux différents niveaux de la scolarité (école primaire, enseignement secondaire, université), et à la formation des enseignants. Ils ont essayé d'approcher la manière dont on peut construire des compétences en litéracie, quelle que soit la discipline envisagée, d'un point de vue linguistique, philosophique, sociologique ou littéraire. Leur regard s'est porté sur les pratiques enseignantes, voire sur les compétences professionnelles des enseignants et la manière dont les étudiants composent avec leur travail d'apprentissage et les raisons qui les conduisent à l'échec ou au succès.

Les différents articles traitent des thèmes suivants :

- L'évaluation

- Quelle évaluation en litéracie ?

- Quels sont les niveaux de litéracie pour les différents groupes d'étudiants ou d'élèves et comment sont-ils évalués?

○ Quels objectifs précis se donne l'enseignement de la litéracie? 


\section{SHS Web of Conferences}

- Equité, différenciation et efficacité

- Quelle corrélation entre les inégalités sociales liées à des habitus litéraciés et les conséquences sur les parcours scolaires?

- Dans quelle mesure écrit et oral peuvent-ils s'enrichir mutuellement en termes d'apprentissage de compétences litéraciées?

- Comment lire, parler, écrire à propos des différents sujets traités à l'école et des disciplines universitaires? 